\title{
Prevalência de distúrbios hematológicos em adolescentes com menorragia
}

\author{
Célio G. Soares, ${ }^{1}$ João Paulo R. Gonçalves, ${ }^{1}$ Denise L. M. Monteiro ${ }^{1,2 *}$
}

\section{Resumo}

Objetivos: conhecer a prevalência e o tipo das principais desordens hematológicas que acometem as mulheres com menorragia, com ênfase no grupo adolescente, para avaliar a necessidade de solicitar testes diagnósticos específicos na investigação de rotina e proporcionar melhor possibilidade terapêutica.

Fonte de dados: revisão sistematizada por meio de ampla pesquisa na literatura médica procurando identificar e extrair informações da literatura, utilizando as bases de dados: Medline (PubMed), SciELO, LILACS, Cochrane e Google Acadêmico. Síntese dos dados: foram identificados 8 estudos sobre prevalência de distúrbios da coagulação em mulheres com menorragia, todos apontando alta prevalência no menacme (10-73\%). Os principais tipos de distúrbios, em ordem decrescente foram: defeitos da função plaquetária (DFP) em 5-67\%, doença de Von Willebrand (DVW) em 5-36\% e deficiência de fator(es) da coagulação(DFC) em 2,5-10\%.Somente 3 estudos incluíram apenas adolescentes, confirmando a alta prevalência também neste grupo: $10,4 \%$, 41\% e 47,8\%, sendo DFP em 5,5-17,7\%, DVW em 5-36\% e DFC12,4\%. A grande variabilidade dos resultados pode ser explicada pela heterogeneidade dos estudos, o que inclui modo e local de recrutamento, características demográficas como etnia e idade, além de critérios para o diagnóstico.

Conclusões: a prevalência de distúrbios da coagulação é bastante elevada, o que reforça a importância de sua investigação em pacientes com menorragia, especialmente nas que apresentam história familiar positiva para sangramento excessivo, levando-se em consideração a avaliação custo-benefício, já que em função do custo e da baixa disponibilidade de determinados exames específicos, torna-se inviável a realização de testes laboratoriais em todas.

Descritores: Menorragia; Hemorragia uterina; Doenças hematológicas; Adolescente.

\section{Abstract \\ Prevalence of bleeding disorders in adolescents with menorrhagia}

Objectives: to know the prevalence and type of major hematologic disorders that affect women with menorrhagia, with emphasis on the adolescent group, in order to evaluate the need to request specific diagnostic tests in routine screening and provide better therapy.

Data sources: an extensive research was carried out in the medical literature, aiming at identifying and extracting information from the literature of the following databases: Medline (PubMed), SciELO, LILACS, Cochrane and Google Scholar.
1. Disciplina de Ginecologia. Departamento Ginecologia/Obstetrícia. Centro Universitário Serra dos Órgãos. Teresópolis, RJ, Brasil.

2. Disciplina de Obstetrícia. Departamento Ginecologia/Obstetrícia. Faculdade de Ciências Médicas. Universidade do Estado do Rio de Janeiro. Rio de Janeiro, RJ, Brasil.

\section{*Endereço para correspondência: Núcleo Perinatal, HUPE, UERJ Avenida Prof. Manuel de Abreu, 500 Rio de Janeiro, RJ. CEP 20550-170. \\ E-mail: denimonteiro2@yahoo.com.br}

Revista HUPE, Rio de Janeiro, 2016;15(1):13-19 doi: $10.12957 /$ rhupe.2016.22356 Recebido em 04/12/2015. Aprovado em 08/12/2015.

Data synthesis: the present study identified 8 studies about prevalence of coagulation disorders in women with menorrhagia, all pointing high prevalence in reproductive age (10-73\%). The main types of disorders, in decreasing order were: platelet function defects (DFP) in 5-67\%, Von Willebrand disease (VWD) disease in 5-36\% and coagulation factor(s) deficiency (DFC) in 2.5-10\%. Only 3 studies were focused exclusively in adolescents, confirming the high prevalence in this group: $10.4 \%, 41 \%$ and $47.8 \%$, with DFP at $5.5-17.7 \%$, DVW at $5-36 \%$ and DFC at $12.4 \%$. The great variability of results can be explained by the heterogeneity of studies, including recruitment forms, demographic characteristics such as ethnicity and age, and diagnosis criteria.

Conclusions: the prevalence of coagulation disorders is high, demonstrating that further research is needed in patients with menorrhagia, especially in those with a family history of excessive bleeding, taking into account the cost-benefit assessment, due to the high cost and low availability of specific tests, it may be non-viable to carry out laboratory tests in all of them.

Keywords: Menorrhagia; Uterine hemorrhage; Hematologic diseases; Adolescent.

\section{Resumen}

\section{Prevalencia de disturbios hematológicos en adoles- centes con menorragia}

Objetivos: determinar la prevalencia y el tipo de los principales desordenes hematológicos que afectan a las mujeres con menorragia, con énfasis en el grupo de adolescentes, para evaluar la necesidad de solicitar pruebas de diagnóstico 


\section{Artigo original}

específico en la investigación de rutina y proporcionar una mejor posibilidad terapéutica.

Fuente de datos: una revisión sistematizada por medio de una amplia investigación en la literatura médica que busque identificar y extraer informaciones de la literatura, utilizando las bases de datos: MedLine, SciELO, LILACS, Cochrane y Google Académico.

Resumen de los datos: fueron identificados 8 estudios sobre prevalencia de los trastornos de la coagulación en mujeres con menorragia, todos de alta prevalencia en el menacme (10-73\%). Los principales tipos de trastornos, en orden decreciente fueron: defectos de la función plaquetaria (DFP) en 5-67\%, Enfermedad de Von Willebrand (EVW) en 5-36\% y deficiencia de factor(es) de la coagulación (DFC) en 2,5-10\%. Solo 3 estudios incluyeron solo adolescentes, lo que confirma la alta prevalencia, también en este grupo:10,4\%, 41\% y 47,8\%,

\section{Introdução}

Osangramento menstrual excessivo ou menorragia é uma queixa frequente em consultórios de ginecologia ou mesmo em serviços de emergência, sendo definido como ciclo menstrual que dura mais de sete dias ou que resulta na perda de $80 \mathrm{ml}$ de sangue ou mais $(\mathrm{A}, \mathrm{B}) .^{1,2}$ Como mensurar a perda menstrual real não é viável na prática clínica, Higham e colaboradores criaram um gráfico pictórico de avaliação do sangramento (PBAC), no qual a pontuação total superior a 100 está relacionada à perda menstrual superior a $80 \mathrm{ml}$. Esse gráfico é a principal ferramenta utilizada, sendo adotada nos estudos analisados nesta revisão para caracterizar a menorragia das pacientes estudadas (A). ${ }^{3}$

As causas de menorragia podem ser divididas em funcionais e orgânicas, sendo que, neste último grupo, os distúrbios da hemostasia representam importante parcela. Estima-se que, a cada ano, aproximadamente $5 \%$ das mulheres em idade reprodutiva procuram ajuda médica devido à menorragia $(\mathrm{A}, \mathrm{B}){ }^{2,4-6}$ As anormalidades hematológicas mais encontradas são: doença de Von Willebrand (DVW), defeitos da função plaquetária (DFP) e deficiência de fator(es) da coagulação (DFC) $(\mathrm{B}, \mathrm{C}) .4,7,8$

O reconhecimento dessas desordens hematológicas requer alto índice de suspeição e testes laboratoriais especializados, por vezes pouco disponíveis, o que pode resultar em um diagnóstico tardio, aumentando, assim, a morbidade (B). ${ }^{5}$ Nas adolescentes, os distúrbios da coagulação comumente não são lembrados dentre os diagnósticos diferenciais e a menorragia é atribuída com frequência à imaturidade do eixo hipotálamohipófise-ovário (B). ${ }^{4,9}$ No entanto, é importante frisar siendo DFP entre 5,5-17,7\%, DVW entre 5-36\% y DFC en 12,4\%. La gran variabilidad de los resultados puede explicarse por la heterogeneidad de los estudios, incluyendo el modo y el lugar de reclutamiento, características demográficas como etnia y edad, además de los criterios para el diagnóstico.

Conclusiones: la prevalencia de los trastornos de la coagulación es bastante alto, lo que refuerza la importancia de su investigación en pacientes con menorragia, especialmente en las que presentan historial familiar positivo para el sangrado excesivo, teniéndose en cuenta la evaluación costo-beneficio, ya que en función del costo y de la baja disponibilidad de determinados exámenes específicos, no es factible llevar a cabo pruebas de laboratorio en absoluto.

Palabras clave: Menorragia; Hemorragia uterina; Enfermedades hematológicas; Adolescente.

que o sangramento menstrual excessivo devido às desordens hematológicas é regularmente espaçado, o que, em geral, não ocorre na imaturidade do eixo. O diagnóstico clínico dos distúrbios da hemostasia consiste na realização de exame físico e obtenção da história clínica detalhada, na qual se devem pesquisar pontos como hematomas frequentes e de reabsorção lenta, hemorragias intensas após injúrias (cirurgias, traumas), epistaxes frequentes e história de sangramentos intensos em outros membros da família. A investigação laboratorial inicial consiste em exames gerais (hemograma e contagens de plaquetas, tempo de protrombina, tempo de tromboplastina parcial ativada, fibrinogênio, tempo de trombina) e em exames específicos, como por exemplo, o antígeno do fator de Von Willebrand (FVW:Ag) e a atividade de cofator de ristocetina (FVW:RCo), utilizados na pesquisa da DVW (B). ${ }^{8}$

Em função do exposto, o objetivo desta revisão sistematizada é conhecer a prevalência e o tipo das principais desordens hematológicas que acometem as mulheres com menorragia, com ênfase no grupo de adolescentes. Como consequência, teremos uma estimativa da real frequência dessas doenças nessa população e então, será possível avaliar a necessidade de solicitar testes diagnósticos específicos na investigação de rotina, visando proporcionar terapêutica mais adequada.

\section{Fontes de dados}

Na confecção desta revisão sistematizada, realizamos ampla pesquisa nos bancos de dados da literatura médica nacional e internacional. As principais bases 
utilizadas foram: SciELO, LILACS, Medline (por meio do PubMed), Cochrane e Google Acadêmico.

No PubMed, foi utilizada a seguinte estratégia de busca: "Adolescent"[Mesh] AND "Hematologic Diseases"[Majr] AND “Uterine Hemorrhage"[Mesh], resultando num total de 94 artigos. Nas demais bases de dados, utilizamos os termos menorragia, menorrhagia, hemorragia uterina, uterine hemorrhage, doenças hematológicas e hematologic diseases, sendo selecionados mais 12 artigos. Após análise individual e conjunta, respeitando os critérios de inclusão e exclusão pré-estabelecidos (Quadro 1), dois revisores selecionaram 22 artigos, dos quais 6 foram excluídos por impossibilidade de acesso ao texto completo. O fluxograma da pesquisa bibliográfica e a seleção dos artigos encontram-se na figura 1 .

Para classificar os trabalhos citados na revisão, utilizamos a classificação proposta pela Associação Médica Brasileira (Quadro 2).

Resultados

As desordens hematológicas foram agrupadas nos três principais grupos (DFP, DVW e DFC), visando melhor visualização dos resultados (Tabela 1).

A prevalência de um ou mais distúrbios da he- mostasia nas mulheres no menacme com menorragia variou entre 10 e $73 \%$, tendo como causas: DFP (5 - 67\%), DVW (5 - 36\%) e DFC (2,5 -10\%).

Somente um estudo ${ }^{13}$ analisou a prevalência da menorragia em pacientes com distúrbio da hemostasia previamente diagnosticado e não a prevalência dos distúrbios da coagulação entre as pacientes com menorragia, como fizeram os autores listados na tabela a seguir, identificando que: $37 \%$ das mulheres com DVW, $72 \%$ das portadoras de DFP e $11 \%$ das com DFC apresentaram menorragia como um dos seus sintomas. Isso demonstra que a menorragia é uma forma de sangramento comum dentre as mulheres com distúrbios da hemostasia, especialmente entre aquelas com DFP $(\mathrm{A}){ }^{13}$

Não foi possível analisar os dados referentes ao grupo de adolescentes de forma isolada em todos os textos revistos, pois a maioria deles não se restringiu

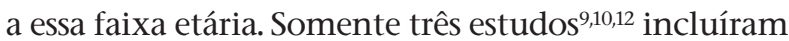
apenas adolescentes, confirmando a alta prevalência, também nesse grupo: 10,4\%, 41\% e 47,8\%. Jayasinghe e colaboradores ${ }^{12}$ encontraram prevalência de DFP de 5,5\% e DVW em 5\% das jovens, Mikhail e colaboradores ${ }^{9}$ descreveram DFP em 7\% e DVW em 36\%, enquanto Se-

Quadro 1. Critérios de inclusão e exclusão.

\begin{tabular}{|l|l|}
\hline \multicolumn{1}{|c|}{ Critérios de inclusão } & \multicolumn{1}{c|}{ Critérios de exclusão } \\
\hline 1. Artigos que abordam distúrbios hemorrágicos; & 1. Estudos que abordam menorragia na gestação/puerpério; \\
\hline $\begin{array}{l}\text { 2. Estudos sobre pacientes com menorragia; } \\
\text { 3. Artigos publicados nos últimos } 10 \text { anos; }\end{array}$ & $\begin{array}{l}\text { 2. Trabalhos exclusivamente sobre determinado distúrbio/ } \\
\text { doença, ou que não citam a prevalência; }\end{array}$ \\
\hline $\begin{array}{l}\text { 4. Artigos publicados nos idiomas: Português, Inglês, Espanhol } \\
\text { e Francês; }\end{array}$ & $\begin{array}{l}\text { 3. Artigos que não abordem a relação entre distúrbios hemor- } \\
\text { rágicos e menorragia; }\end{array}$ \\
$\begin{array}{l}\text { 5. Estudos com foco na determinação da prevalência dos distúr- } \\
\text { bios hemorrágicos; }\end{array}$ & \\
\hline \begin{tabular}{l} 
6. Foco em mulheres nom foco em terapenticas específicas/méto- \\
\hline
\end{tabular}
\end{tabular}

\section{Quadro 2. Grau de recomendação e força de evidência.}

\begin{tabular}{|c|l|}
\hline A & Estudos experimentais ou observacionais de melhor consistência (metanálises ou ensaios clínicos randomizados). \\
\hline B & $\begin{array}{l}\text { Estudos experimentais ou observacionais de menos consistência (outros ensaios clínicos não randomizados ou estudos } \\
\text { observacionais ou estudos caso-controle). }\end{array}$ \\
C & Relatos ou séries de casos (estudos não controlados). \\
\hline D & Opinião desprovida de avaliação crítica, baseada em consensos, estudos fisiológicos ou modelos animais. \\
\hline
\end{tabular}




\section{Artigo original}

Tabela 1. Distribuição da prevalência de desordens hematológicas em pacientes com menorragia.

\begin{tabular}{|c|c|c|c|c|c|}
\hline Autor/Ano & Tipo de Estudo & Amostra & Idade & $\begin{array}{c}\text { Prevalência des. } \\
\text { hematológicas }\end{array}$ & $\begin{array}{c}\text { Causas } \\
\text { des. hematológicas }\end{array}$ \\
\hline $\begin{array}{l}\text { Seravalli V et al. } \\
\qquad(2013)^{10}\end{array}$ & $\begin{array}{l}\text { Corte Transversal } \\
\text { (B) }\end{array}$ & 113 & $13,5 \pm 2,8$ & $113 / 54(47,8 \%)$ & 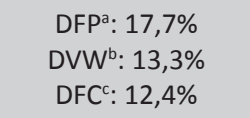 \\
\hline $\begin{array}{l}\text { Philipp CS et al. } \\
\qquad(2011)^{4}\end{array}$ & $\begin{array}{l}\text { Corte Transversal } \\
\text { (B) }\end{array}$ & 217 & 18 a 50 & $154 / 217(71 \%)$ & $\begin{array}{l}\text { DFP: } 58,5 \% \\
\text { DVW: } 5,1 \% \\
\text { DFC: } 5,1 \%\end{array}$ \\
\hline $\begin{array}{l}\text { Miller CH et al. } \\
\qquad(2011)^{11}\end{array}$ & $\begin{array}{l}\text { Corte Transversal } \\
\text { (B) }\end{array}$ & 232 & 18 a 50 & $169 / 232(73 \%)$ & $\begin{array}{l}\text { DFP: } 62,5 \% \\
\text { DVW: } 6 \% \\
\text { DFC: } 10 \%\end{array}$ \\
\hline $\begin{array}{l}\text { Philipp CS et al. } \\
(2008)^{5}\end{array}$ & $\begin{array}{l}\text { Coorte } \\
\text { (B) }\end{array}$ & 146 & 13 a 53 & $102 / 146(70 \%)$ & $\begin{array}{l}\text { DFP: } 67 \% \\
\text { DVW: } 8 \% \\
\text { DFC: } 7 \%\end{array}$ \\
\hline $\begin{array}{l}\text { Mikhail S et al. } \\
(2007)^{9}\end{array}$ & $\begin{array}{l}\text { Corte Transversal } \\
\text { (B) }\end{array}$ & 61 & 11 a 19 & $25 / 61$ (41\%) & $\begin{array}{l}\text { DFP: } 7 \% \\
\text { DVW: } 36 \%\end{array}$ \\
\hline $\begin{array}{l}\text { Philipp CS et al. } \\
\qquad(2005)^{6}\end{array}$ & $\begin{array}{l}\text { Coorte } \\
\text { (B) }\end{array}$ & 115 & 13 a 55 & $54 / 115$ (47\%) & $\begin{array}{l}\text { DFP: } 43 \% \\
\text { DVW: } 7 \% \\
\text { DFC: } 5 \%\end{array}$ \\
\hline $\begin{array}{l}\text { Jayasinghe } Y \text { et al. } \\
(2005)^{12}\end{array}$ & $\begin{array}{l}\text { Corte Transversal } \\
\text { (B) }\end{array}$ & 106 & 9 a 19 & $11 / 106(10 \%)$ & $\begin{array}{l}\text { DFP: } 5,5 \% \\
\text { DVW: } 5 \%\end{array}$ \\
\hline $\begin{array}{c}\text { Trasi AS et al. } \\
(2004)^{2}\end{array}$ & $\begin{array}{l}\text { Coorte } \\
\text { (B) }\end{array}$ & 120 & 18 a 35 & $23 / 120$ (19\%) & $\begin{array}{l}\text { DFP: } 5 \% \\
\text { DVW: } 11 \% \\
\text { DFC: } 2,5 \%\end{array}$ \\
\hline
\end{tabular}

aFP - Defeitos da Função Plaquetária; 'bVW - Doença de Von Willebrand; 'DFC - Deficiência de Fator(es) de Coagulação.

ravalli e colaboradores ${ }^{10}$ relataram a maior prevalência de distúrbios da coagulação entre adolescentes com menorragia (47,8\%), sendo: DFP em 17,7\%, DVW em 13,3\% e DFC em 12,4\%, dados que confirmam a importância de se pensar na possibilidade desses distúrbios ao se atender uma adolescente com menorragia.

A grande variabilidade dos resultados pode ser explicada pela heterogeneidade dos estudos, o que inclui modo e local de recrutamento, características demográficas como etnia e idade, além de critérios para o diagnóstico.

\section{Discussão}

A menorragia é uma queixa comum, relatada por $30 \%$ das mulheres em idade reprodutiva, e anualmente $5 \%$ das mulheres procuram atendimento médico por sangramento menstrual excessivo (B). ${ }^{6}$

Nas pacientes com distúrbios hemorrágicos, a menorragia pode se apresentar desde a menarca e, não raro, faz com que elas necessitem de internação hospitalar ainda no primeiro ano após esse marco (B). ${ }^{13}$ No entanto, Seravalli e colaboradores, ${ }^{10}$ ao analisarem um grupo de adolescentes, não encontraram diferença significativa na ocorrência de distúrbios hemorrágicos entre as pacientes que apresentaram menorragia nos dois primeiros anos pós-menarca em comparação com aquelas que apresentaram mais tardiamente, demonstrando que a menorragia precoce não é um bom preditor clínico de distúrbio hemorrágico (B). ${ }^{10}$

O reconhecimento do diagnóstico dessas pacientes não é tarefa fácil, podendo levar anos, o que contribui para o aumento da morbidade $(\mathrm{A}, \mathrm{B}))^{2,4,11,12}$

Nossa proposta inicial era avaliar a prevalência da menorragia com ênfase no grupo de adolescentes. No entanto, a maioria dos artigos encontrados não se limitou a essa faixa etária e incluiu mulheres no menacme, mas com maior idade, constituindo a maior limitação do nosso estudo. Phillip e colaboradores ${ }^{6}$ analisaram a prevalência de distúrbios hemorrágicos por faixa etária e concluíram que adolescentes, mulheres adultas e na perimenopausa que se apresentam com menorragia sem causa aparente possuem a mesma probabilidade de ter distúrbio hemorrágico subjacente (B). ${ }^{6}$ Dessa forma, a impossibilidade de avaliar somente o grupo 
de adolescentes não invalida os resultados encontrados.

Nesta revisão, encontramos prevalência de distúrbios da coagulação entre as mulheres com menorragia variando entre 10 e 73\%. Essa grande variabilidade na prevalência pode ser devida ao contexto no qual a população de estudo estava inserida. Os estudos que avaliaram mulheres que estavam recebendo cuidados em centros mais especializados, como os centros de tratamento de hemofilia, tenderam a ter prevalências mais elevadas, o que reforça a importância de centros desse tipo no diagnóstico dessas desordens (A).9,11,13 Antes de serem selecionadas para participação nos estudos, as pacientes passaram por avaliação clínica com o intuito de excluir aquelas que apresentavam outras condições que pudessem ser responsáveis pela menorragia, como miomas e uso de drogas com efeitos sobre a coagulação. Essa seleção de pacientes contribuiu para a ocorrência de elevada prevalência em alguns estudos (A),9,11,13

Além disso, é importante salientar que diversos métodos laboratoriais estão disponíveis para o diagnóstico de uma mesma doença, o que também pode contribuir para essa variabilidade da prevalência, já que esses métodos possuem diferentes valores de sensibilidade/especificidade, alguns, inclusive, podem ter seus resultados alterados a depender da fase do ciclo menstrual em que são realizados (A).9,11

Essa grande discrepância entre os valores de prevalência diminui a confiabilidade dos dados e dificulta a análise crítica. Portanto, ainda há a necessidade de melhor determinação da real prevalência dos distúrbios hemorrágicos nessa população.

A DVW é um distúrbio da hemostasia adquirido ou hereditário, sendo esta última forma, a mais comum. Nessa doença há um defeito quantitativo ou qualitativo do fator de Von Willebrand (FVW), o qual age ligando-se ao colágeno no subendotélio e nas plaquetas, auxiliando a formação do tampão plaquetário no local da hemorragia. Ele também se liga ao fator VIII e, dessa forma, protege-o da atividade proteolítica do plasma $(\mathrm{A}){ }^{14}$

Essa desordem é classificada em três tipos (1, 2 e 3). Nos tipos 1 e 3 há alteração quantitativa do FVW, enquanto no tipo 2 há alteração funcional. Os principais exames na investigação da DVW são: o estudo funcional do FVW por meio da sua atividade de cofator de ristocetina, o teste imunológico para o FVW e a avaliação da função do fator VIII (A). ${ }^{6,14}$ Em nossa revisão, a DVW foi a segunda patologia mais encontrada com prevalência variando entre 5,1-48\%.

A DVW é menos encontrada entre as mulheres ne- gras. Nesse grupo de mulheres foram observados níveis maiores de antígeno de FVW e atividade do fator VIII que aqueles encontrados entre as mulheres brancas. Estudos que avaliaram a população norte-americana, a qual apresenta significativa proporção de mulheres negras, têm encontrado frequências menores de DVW que os estudos europeus. Miller e colaboradores avaliaram um grupo de mulheres norte-americanas e observaram que a DVW ocorreu duas vezes mais entre as brancas (B). ${ }^{6,11}$

Os DFP formam um grupo complexo e heterogêneo de doenças, as quais podem interferir em diferentes fases da adesão/agregação plaquetária. As plaquetas se aderem nas regiões onde o endotélio foi lesado e dão início à formação do tampão plaquetário, quando então são ativadas e liberam substâncias presentes em seus grânulos, estimulando a adesão de mais plaquetas (A). ${ }^{15}$

O diagnóstico dessas desordens geralmente é complicado devido à falta de testes laboratoriais uniformes e às dificuldades na interpretação e na realização desses exames. Dessa forma, torna-se difícil determinar com precisão a prevalência dos distúrbios plaquetários, podendo essa ter sido subvalorizada em estudos que não avaliaram a função plaquetária sistematicamente (A,B). ${ }^{4,6,11,12,15}$

Os principais testes laboratoriais disponíveis são: agregometria plaquetária, analisador da função plaquetária (PFA-100), microscopia eletrônica de plaquetas, citometria de fluxo e tromboelastrografia (A). ${ }^{11,12}$

Nesta revisão, os DFP foram o grupo de doenças mais encontrado, com prevalência variando entre 5-67\%. Esse grupo também foi o que apresentou maior variabilidade na prevalência, o que possivelmente se deve às dificuldades técnicas na realização dos exames laboratoriais citados acima (A).9,11 Dessa forma, o desenvolvimento de testes mais específicos da função plaquetária deve ser o objetivo de pesquisa futura (A). ${ }^{11}$

As mulheres negras apresentam frequência maior de DFP. Dessa forma, a característica étnica de cada população também é fator que interfere nos níveis de prevalência (A). ${ }^{11}$

Os fatores de coagulação são proteínas plasmáticas que atuam no processo de formação do coágulo sanguíneo. Embora com frequências mais baixas do que as relatadas para disfunção plaquetária e DVW, a DFC também é outro grupo de distúrbio hemorrágico comumente encontrado nessas mulheres, particularmente a deficiência dos fatores V, VII e IX (B). ${ }^{6}$ Os fatores geralmente dosados são: II, V, VII, VIII, IX, X, XI e XII. Observamos que a prevalência de DFC variou entre 2,5a 


\section{Artigo original}

10\%, sendo o grupo menos prevalente em nosso estudo.

As pacientes com distúrbios hemorrágicos, além da menorragia, apresentam com frequência história de sangramentos em outros sítios. Mikhail e colaboradores ${ }^{9}$ encontraram a seguinte distribuição de problemas hemorrágicos entre as mulheres com distúrbios hematológicos: epistaxe (24\%), equimoses (32\%), sangramento gengival (28\%), sangramento excessivo após procedimentos invasivos (12\%) e história familiar de hemorragia (40\%). No entanto, é importante frisar que a menorragia, em alguns estudos, foi o único sintoma hemorrágico em 20\% das mulheres com distúrbios hematológicos $(\mathrm{A}){ }^{7,13}$ Outro achado comum é a presença de anemia ferropriva (A). ${ }^{4}$

Embora os sangramentos em outros sítios sejam sintomas típicos das mulheres com distúrbios da hemostasia, para a maioria dos autores, esses sintomas não foram importantes preditores clínicos de doença hemorrágica (A)., ${ }^{913}$ Jayasinghe e colaboradores ${ }^{12}$ definiram a história familiar de equimose/hematoma ou hemorragia como única variável clínica com valor preditivo para distúrbio de sangramento ( $p=0,03)$. É interessante notar que, muitas vezes, a história de menorragia se perpetua em sucessivas gerações de mulheres de uma mesma família e essas não procuram ajuda médica por considerarem uma norma na família (B). ${ }^{2}$

O manejo dessas mulheres requer que, primeiramente, seja feita a avaliação ginecológica minuciosa para descartar outras causas de menorragia que não os distúrbios hemorrágicos. Devem-se levar em consideração a idade, as condições clínicas e o desejo de engravidar dessas pacientes (A). ${ }^{4}$

Classicamente, métodos hormonais têm sido utilizados para o controle do sangramento menstrual nessas pacientes. Os anticoncepcionais orais (ACO) em regime contínuo se mostraram eficazes em diminuir a perda menstrual nas mulheres com distúrbios hemorrágicos, especialmente naquelas com DVW, nas quais também possivelmente aumentam os níveis séricos do FVW e fator VIII (A).,13 O dispositivo intrauterino de levonorgestrel também é um método que se mostrou eficaz no controle da menorragia (A), ${ }^{12}$ podendo, inclusive, ser usado em adolescentes, de acordo com os critérios de elegibilidade da Organização Mundial de Saúde (A). ${ }^{16}$

Os agonistas do GnRH também são outro grupo de fármacos descritos que se mostraram eficazes no controle da menorragia; no entanto, seu uso a longo prazo não é adequado devido a efeitos adversos importantes causados pelo hipoestrogenismo que incluem, dentre eles, a redução da densidade óssea $(\mathrm{A}){ }^{13}$
Para as mulheres que não tiveram controle satisfatório da menorragia com os métodos hormonais ou que desejam gestar, terapias com medicamentos antifibrinolíticos (ácido aminocapróico e ácido tranexâmico), concentrados de fatores de coagulação e desmopressina (DDAVP) podem ser utilizados (A).,13 Para mulheres com prole constituída, a ablação endometrial tem sido opção cirúrgica menos invasiva que a histerectomia, demostrando ter eficácia semelhante (A). ${ }^{1}$

A avaliação hematológica de todas as mulheres com menorragia sem causa aparente que se apresentam no serviço de saúde é impraticável dentro do contexto de saúde pública. Assim, métodos que facilitem o reconhecimento das pacientes com distúrbios hematológicos subjacentes seriam de grande valia. $\mathrm{O}$ método proposto por Phillip e colaboradores, ${ }^{6}$ que consiste de oito questões diretas para detectar distúrbios da coagulação, demonstrou sensibilidade de 89\% e valor preditivo positivo de $72 \%$, revelando-se, assim, como excelente ferramenta para triagem. Quando uma pontuação do gráfico PBAC de 185 ou mais, ou o nível de ferritina sérica $<20 \mathrm{ng} / \mathrm{ml}$ foram combinados com essa ferramenta, conseguiu-se elevar a sensibilidade para $95 \%(\mathrm{~A}){ }^{4}$

O gráfico PBAC possui elevada sensibilidade/especificidade para menorragia, sendo utilizado para caracterizá-la de forma mais objetiva. Entretanto, ele não é um método prático, pois requer a avaliação de todo ciclo menstrual para se chegar ao resultado, inviabilizando seu uso em emergências. Além disso, não houve diferença significativa na prevalência de distúrbios da hemostasia quando o valor de PBAC maior que 100 era requisito para inclusão no estudo, demonstrando, assim, não ser bom método de triagem (A).,11

\section{Conclusões}

Neste estudo, observamos que a prevalência de distúrbios da coagulação foi bastante elevada, o que reforça a importância de sua investigação em pacientes com menorragia, visando proporcionar terapêutica mais adequada. A discrepância das prevalências entre alguns estudos demonstra a heterogeneidade dos estudos, necessitando mais pesquisas nessa área para se chegar a dados mais confiáveis da real prevalência desses distúrbios nessas mulheres. As doenças mais encontradas em ordem decrescente foram: DFP, DVW e DFC.

Dessa forma, no atendimento a uma paciente apresentando menorragia, devemos sempre ter em mente a possibilidade da presença de distúrbio da coagulação, principalmente naquelas com história familiar positiva 
para hemorragia. Contudo, a avaliação dessas mulheres deve levar em consideração a relação custo-benefício, pois é inviável a realização de testes laboratoriais em todas. A aplicação de métodos de triagem que se mostrem eficientes em demonstrar quais mulheres têm maior probabilidade de possuir doenças da coagulação facilitaria a seleção das que necessitam de avaliação hematológica mais aprimorada e de eventual encaminhamento a centro especializado.

\section{Referências}

1. James AH. Women and bleeding disorders. Haemophilia. 2010;16 Suppl 5:160-7. http://dx.doi.org/10.1111/j.13652516.2010.02315.x.

2. Trasi SA, Pathare AV, Shetty SD, et al. The spectrum of bleeding disorders in women with menorrhagia: a report from Western India. Ann Hematol. 2005;84(5):339-42.

3. Higham JM, OBrien PM, Shaw RW. Assessment of menstrual blood loss using a pictorial chart. Br J Obstet Gynaecol. 1990;97:734-9.

4. Philipp CS, Faiz A, Heit JA, et al. Evaluation of a screening tool for bleeding disorders in a US multisite cohort of women with menorrhagia. Am J Obstet Gynecol. 2011;204(3):209.e1-7. http://dx.doi.org/10.1016/j.ajog.2010.10.897.

5. Philipp CS, Faiz A, Dowling NF, et al. Development of a screening tool for identifying women with menorrhagia for hemostatic evaluation. Am J Obstet Gynecol. 2008;198(2):163.e1-8. http:// dx.doi.org/10.1016/j.ajog.2007.08.070.

6. Philipp CS, Faiz A, Heit JA, et al. Evaluation of a screening tool for bleeding disorders in a US multisite cohort of women with menorrhagia. Am J Obstet Gynecol. 2011;204(3):209.e1-7. doi: 10.1016/j.ajog.2010.10.897.

7. Weiss JA. Just heavy menses or something more?
Raising awareness of von Willebrand disease. Am J Nurs. 2012;112(6):38-44. http://dx.doi.org/10.1097/01. NAJ.0000415122.54111.f4.

8. Halimeh S. Menorrhagia and bleeding disorders in adolescent females. Hamostaseologie. 2012;32(1):45-50. http://dx.doi. org/10.5482/ha-1181. 2011.

9. Mikhail S, Varadarajan R, Kouides P. The prevalence of disorders of haemostasis in adolescents with menorrhagia referred to a haemophilia treatment centre. Haemophilia. 2007;13(5):627-32.

10. Seravalli V, Linari S, Peruzzi E, et al. Prevalence of Hemostatic Disorders in Adolescents with Abnormal Uterine Bleeding. J Pediatr Adolesc Gynecol. 2013;26:285e289. http://dx.doi. org/10.1016/j.jpag.2013.06.003.

11. Miller $\mathrm{CH}$, Philipp CS, Stein SF, et al. The spectrum of haemostatic characteristics of women with unexplained menorrhagia.Haemophilia. 2011;17(1):e223-9. http://dx.doi. org/10.1111/j.1365 2516.2010.02382.x.

12. Jayasinghe $Y$, Moore $P$, Donath $S$, et al. Bleeding disorders in teenagers presenting with menorrhagia. Aust $\mathrm{N} Z \mathrm{~J}$ Obstet Gynaecol. 2005;45(5):439-43.

13. Chi C, Pollard D, Tuddenham EG, et al. Menorrhagia in adolescents with inherited bleeding disorders. J Pediatr Adolesc Gynecol. 2010;23(4):215-22. http//10.1016/j.jpag.2009.11.008

14. Brasil. Ministério da Saúde. Secretaria de Atenção à Saúde. Departamento de Atenção Especializada. Manual de diagnóstico e tratamento da doença de Von Willebrand. Série A. Normas e Manuais Técnicos. Brasília; 2006.

15. Sokkary NA, Venkateswaran L, Dietrich JE, et al. Platelet function disorders and menorrhagia in adolescents: a review of laboratory diagnosis. J Pediatr Adolesc Gynecol. 2012;25(4):233-7. http://dx.doi.org/10.1016/j.jpag.2012.01.004.

16. World Health Organization. Medical eligibility criteria for contraceptive use. 4 ed. Genebra, 2010. 121 p. Disponível em http:// www.who.int/reproductivehealth/ publications/family_planning/9789241563888/en/. Acesso em 20Abr2013. 